\title{
Asymmetric Acral Spared Phenomenon Related to Systemic Anticancer Therapies
}

\author{
Robert Baran $^{\text {a }}$ Caroline Robert $^{\mathrm{b}}$ Vincent Sibaud ${ }^{\mathrm{c}}$ \\ ${ }^{a}$ Nail Disease Center, Cannes, France; ${ }^{b}$ Department of Oncology and Dermatology, Gustave Roussy Institute, \\ Villejuif, France; ' ${ }^{C}$ Department of Oncodermatology, Claudius Regaud Institute, Cancer University Institute, Toulouse \\ Oncopole, Toulouse, France
}

\section{Established Facts}

- Unilateral acral drug-induced toxicities have rarely been reported.

- The integrity of central or peripheral nerves seems to be a substantial factor for developing nail unit alterations secondary to docetaxel.

\section{Novel Insights}

- Three new observations allow us to confirm that acral drug-induced toxicities require the integrity of the central and peripheral nervous system.

- The mechanism of taxane-related onycholysis is now better understood.

- The asymmetric acral phenomenon is not only observed with taxanes but also with sorafenib and adriamycin.

\section{Keywords}

Unilateral acral changes - Anticancer therapies - Taxanes · Anticox $2 \cdot$ Onycholysis · Hand-foot syndrome - Reaction sparing a limb unilaterally

\section{Abstract \\ We have observed three patients on anticancer therapies presenting with asymmetric acral spared phenomenon and found six identical cases in the literature. All of them had common features, an alteration of the peripheral nerves af- fecting a limb unilaterally that was spared by a hand-foot}

๑) 2018 S. Karger AG, Basel

E-Mail karger@karger.com www.karger.com/sad syndrome or hand-foot skin reaction. A drug-induced neurotropic effect sounds logical for developing such alterations with specific chemotherapeutic agents (taxanes, capecitabine), while we have not found good explanations concerning the multikinase inhibitor sorafenib nor the topoisomerase inhibitor adriamycin. Nevertheless, we know that clinical manifestations of many inflammatory diseases need intact neural components.

(c) 2018 S. Karger AG, Basel

All authors contributed equally to this work.

Robert Baran, MD 


\section{Introduction}

Acral and nail unit toxicities induced by chemotherapy and targeted therapies have been extensively reported $[1,2]$. Nevertheless, there are very few papers recording unilateral acral changes related to anticancer therapies. We have observed three patients on anticancer therapies presenting with asymmetric acral spared phenomenon.

\section{Case Reports}

Case 1

In 2010 we examined a 48 -year-old male treated with the multikinase inhibitor sorafenib (400 mg, twice a day) for cervical sarcoma infiltrating the axillary fossa and the left brachial plexus leading to palsy of the left upper limb. Interestingly he presented a hand-foot (HF) skin reaction (grade 2/3) of the right hand and both feet associated with dysesthesia and erythema areas predominantly involving the proximal nail folds and the dorsum of the joints of the digits, sometimes with bullae. These sorafenib-related lesions spared the paretic left hand (Fig. 1).

\section{Case 2}

In 2016 we treated a 66-year-old man for a squamous cell carcinoma of the lung associated with metastatic lesions (liver and bone). As medical background, a car crash 15 years prior had left him with a partial peripheral palsy of the upper left limb. Weekly paclitaxel regimen $\left(80 \mathrm{mg} / \mathrm{m}^{2}, 1\right.$-hour infusion) was started.

After 5 cycles of chemotherapy, the patient developed a progressive painful grade 3 onycholysis with purulent discharge and subungual abscesses, involving all the fingernails of the right hand. Surprisingly, a relative respect of the left hand was noted. An inflammatory erythema of the right dorsal hand, suggestive of a
PATEO (periarticular thenar erythema with onycholysis) syndrome was also associated with a sparing of the left dorsal hand [1, 3] (Fig. 2). A Staphylococcus aureus superinfection was diagnosed and was treated with oral antibiotics, associated with nail plate clipping and partial nail avulsion together with nail bed cleaning.

\section{Case 3}

A 50-year-old man presented with a metastatic renal cell carcinoma and a total right hemiplegia, secondary to brain metastases with hemorrhagic suffusions. He was treated with oral sorafenib (400 mg, twice a day) and developed a unilateral grade 2 HF skin reaction involving the left plantar area, with typical localized hyperkeratotic lesions and subungual hyperkeratosis. The lesions totally spared the contralateral sole (Fig. 3).

\section{Previously Reported Cases}

(a) In 2001, Wasner et al. [4] described unilateral nail changes secondary to docetaxel treatment. Subungual hemorrhages, painful paronychia, and onycholysis occurred on both feet and the left hand, sparing the right upper limb, which had a complete peripheral palsy and sensory loss of the distal right arm. This 62-year-old woman suffered from a locally advanced breast cancer, associated with a right brachial plexus infiltration. Neurophysiological testing indicated severe small and large fiber denervation.

(b) In 2006, Bird et al. [5] observed a 53-year-old woman with metastatic breast cancer presenting with right arm pain and weakness. Clinically she had a right supraclavicular fossa mass, complete brachial plexopathy, and Horner's syndrome. The patient started a chemotherapeutic regimen containing adriamycin and cyclophosphamide with a partial response after three cycles associated with right arm function improvement. She developed white transverse lines on the nails of her left hand after each cycle of chemotherapy. Interestingly, the nails of her right hand were totally spared.

(c) In 2009 Truchuelo et al. [6] reported a 48-year-old woman diagnosed in 1999 with a HER-2-positive breast cancer and treated

Fig. 1. Unilateral hand-foot syndrome.

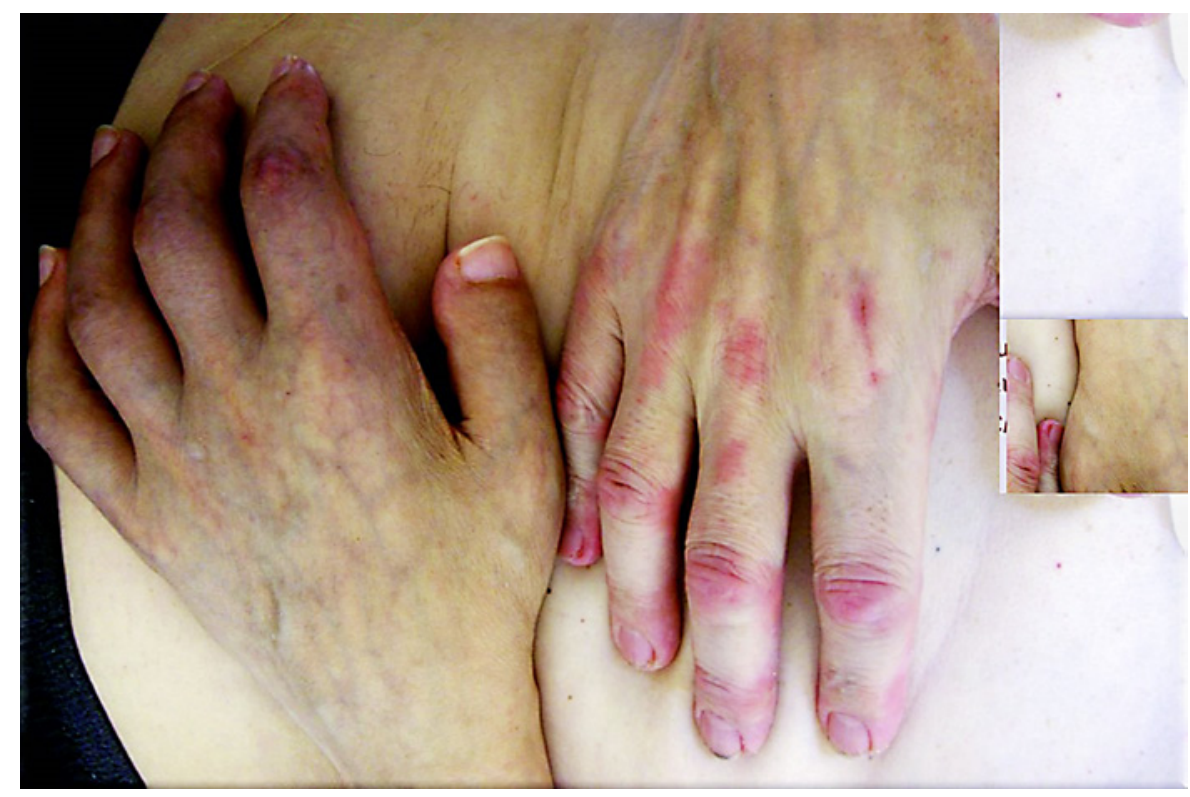


surgically, in combination with adjuvant radiochemotherapy plus hormonotherapy. In 2003, liver, bone, and lung metastases were detected. Docetaxel plus trastuzumab were initiated and produced a complete radiological response of the liver metastases. In 2005, additional metastases affecting liver, bone, and brain were detected and the patient underwent treatment with radiotherapy, trastuzumab, and vinorelbine, with poor tolerance. This chemotherapeutic regimen was replaced by weekly administration of paclitaxel $\left(80 \mathrm{mg} / \mathrm{m}^{2}\right)$, combined with carboplatin. During the following 3 months, she developed progressive paclitaxel-induced onycholysis and leukonychia affecting the 20 nails. Carboplatin was replaced by trastuzumab owing to an anaphylactic reaction and in June 2006 capecitabine was added due to clinical progression. In December 2006, a progressive left upper body hemiparesis developed that involved the left hand. A computed tomography brain scan showed a metastasis located to the right internal capsule. In December 2007, capecitabine was replaced by docetaxel plus lapatinib due to grade $3 \mathrm{HF}$ syndrome and clinical progres- sion. Six months after the introduction of docetaxel, the patient developed onycholysis and leukonychia affecting all nails except those of the left paretic hand.

(d) In November 2009, a 53-year-old Brazilian colored man presented with a $4.5-\mathrm{cm}$ tumor in the descending colon identified by a colonoscopy [7]. One month later, the patient underwent a left colectomy, which found a $\mathrm{pT} 3 \mathrm{pN} 2$ moderate differentiated adenocarcinoma of the sigmoid. Thorax computed tomography also showed multiple bilateral lung nodules. The patient had a history of a right cerebral vascular accident in April 2008, which left him with important motor sequelae. He had hemiparesis of the left upper and lower limbs, more pronounced in his left arm. The patient was treated with capecitabine $1 \mathrm{~g} / \mathrm{m}^{2}$ orally twice a day $(6$ tablets a day) for 14 consecutive days every 3 weeks. After the first cycle of capecitabine, the patient developed grade 2 unilateral HF syndrome only in the right (normal) palm. The left hand had intact skin. As the clinical condition deteriorated, chemotherapy was discontinued.

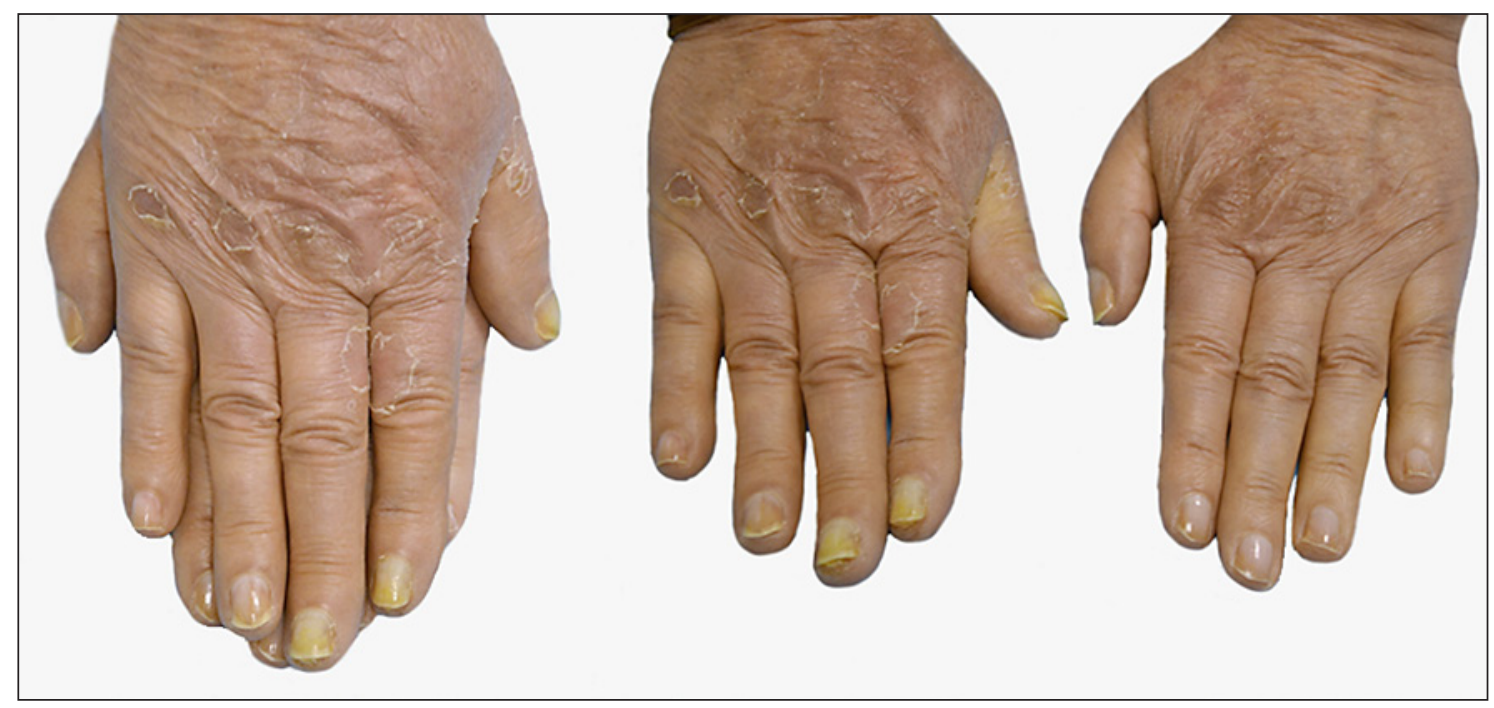

Fig. 2. PATEO syndrome with onycholysis, associated with a sparing of the left dorsal hand.

Fig. 3. Unilateral hand-foot skin reaction involving the right plantar area with subungual hyperkeratosis.

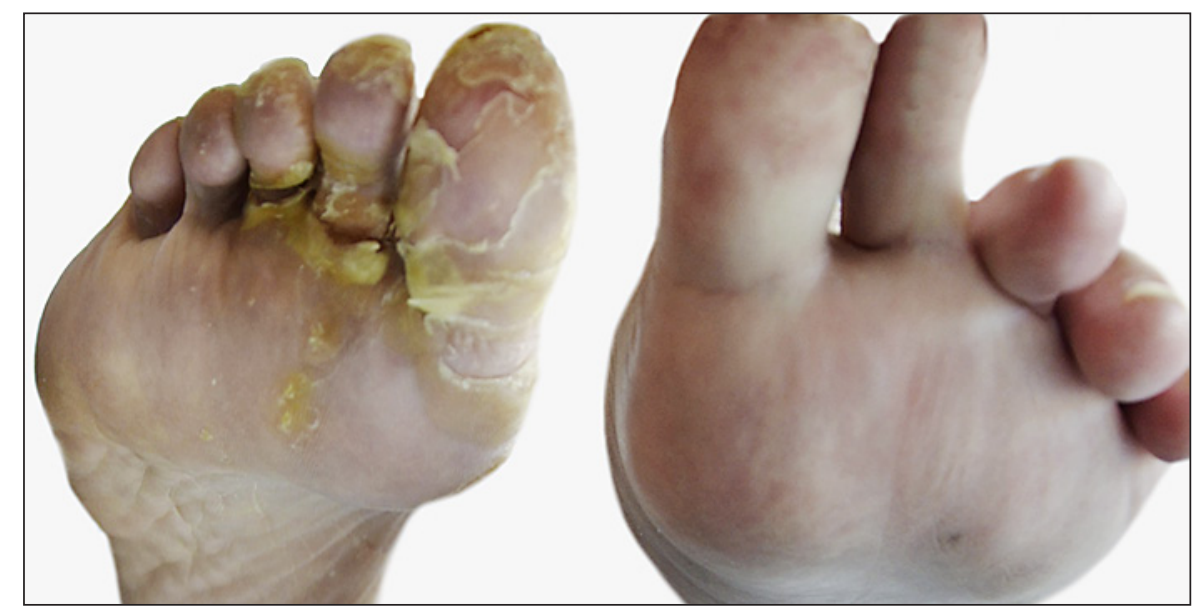


(e) A 65-year-old Turkish Cypriot male patient was diagnosed with advanced gastric cancer [8]. Liver and lung metastases were found on computed tomography scan [8]. His past medical history included hypertension, non-insulin-dependent diabetes mellitus, and cerebral infarction with right hemiplegia. Administration of docetaxel, cisplatin, and infusional 5-fluorouracil was begun with palliative intent. Three months later, liver and lung lesions progressed and single-agent capecitabine administration was started at a dose of $1,000 \mathrm{mg} / \mathrm{m}^{2} /$ day twice a day on days $1-14$ every 21 days. Ten days after the fourth cycle, he suffered from pain, numbness, and reddening in his left palm and left sole. On physical examination, he had unilaterally erythematous changes and skin scaling on his left sole and palm. The case was considered as grade II HF syndrome related to capecitabine. His extremities had muscle atrophy, spastic rigidity, and atrophic skin changes secondary to hemiplegia.

(f) A 60-year-old man with a history of cerebral infarction [9] was subsequently diagnosed to have multiple liver metastases of advanced descending colon cancer, based on the findings of computed tomography. His physical examination showed hemiplegia of the right upper and lower limbs. He was treated with palliative chemotherapeutic regimen consisting of capecitabine, oxaliplatin, and bevacizumab. After two cycles of this chemotherapy, HF syndrome prominently developed in the palm of his left hand.

\section{Discussion}

All these case reports, where drug-induced toxicity of the nails and extremities did not occur in areas with associated neurological alteration, shed some light on the mechanisms of such drug-induced adverse events, in particular, taxane-induced onycholysis, whose pathophysiology was not clearly established [2].

It has been recently observed that taxane-related onycholysis is directly correlated to the duration of the infusion, which could suggest a direct effect resulting from a cytotoxic damage to the nail bed epithelium with secondary loss of adhesion of the nail plate to the nail bed $[1,3]$. An intrinsic antiangiogenic activity of taxanes has also been postulated as well as a phototoxic mechanism. However, etiopathogenesis remains uncertain as none of these hypotheses have been clearly documented. Consequently, this may suggest that a drug-induced neurotropic effect is the most attractive explanation. Indeed, the integrity of peripheral nerves seems to be a substantial factor for developing nail unit alterations secondary to docetaxel [4]. Two neurotropic mechanisms can be envisioned: taxoids may activate nociceptive $\mathrm{C}$-fibers that cause neurogenic inflammation by releasing prostaglandins from sympathetic postganglionic terminals.

This is in line with recent data by Azimi et al. [10], who have demonstrated that the contribution of the nervous system to inflammation in general and inflammatory skin diseases in particular has been underestimated. It is now apparent that an intact neural component is required for the conventional clinical manifestations of many inflammatory skin diseases. The same authors identified 19 cases of preexisting skin lesions (eczema, psoriasis, scleroderma, rosacea, bullous pemphigoid...) with a near or complete resolution in the areas supplied by nerves that were injured or compromised.

It is interesting to remember the very illustrative case reported by Wang and Tsai [11] of a 67-year-old man seen for aggravation of his psoriasis in recent months without a definitive cause, but with a sparing of his left lower limb. He was otherwise healthy except for poliomyelitis, which had affected the ipsilateral limb since childhood. The psoriatic lesions gradually resolved after treatment with oral methotrexate and acitretin in addition to topical calcipotriol. Of note, even joint sparing was also observed in a patient with unilateral psoriatic arthritis and a long-standing hemiplegia [12]. Finally, it has been hypothesized that sparing of psoriasis in autografts was related to a potential "denervation" of the grafted skin [13].

\section{Conclusion}

The asymmetric acral spared phenomenon suggests that the integrity of acquired central or peripheral nerves is needed for the development of anticancer toxicities. More importantly, it may open new therapeutic avenues and lead to the development of new supportive measures (e.g., anticox 2) which may reduce the neurogenic inflammation produced by some anticancer therapies [14].

\section{Statement of Ethics}

The patients have given their informed consent for the presentation of the case.

\section{Disclosure Statement}

Robert Baran: Bailleul. Caroline Robert: BMS, MSD, Roche, Pierre Fabre, Novartis, Amgen. Vincent Sibaud: BMS, Roche, Pierre Fabre. 


\section{References}

-1 Robert C, Sibaud V, Mateus C, Vershoore M, Charles G, Lanoy E, Baran R: Nail toxicities induced by systemic anticancer treatments. Lancet Oncol 2015;16:e181-e189.

-2 Sibaud V, Leboeuf NR, Roche H, Belum VR, Gladieff L, Deslandres M, Montastruc M, Eche A, Vigarios E, Dalenc F, Lacouture ME: Dermatological adverse events with taxane chemotherapy. Eur J Dermatol 2016;26:427443.

3 Sibaud V, Baran R, Piraccini BM, Lacouture M: Drug-induced nail disorders. Anticancer therapies; in Baran-Dawber's Diseases of the Nails and Their Management, 5th edition. Chapter $11 \mathrm{~b}$ (In press).

$\checkmark 4$ Wasner G, Hilpert F, Baron R, Pfisterer J: Clinical picture: nail changes secondary to docetaxel. Lancet 2001;357:910.
Bird BR, Elfiki T, Tucker O, O’Reilly S: Unilateral nail changes secondary to adriamycin: the protective effect of brachial plexopathy. Ann Oncol 2006;17:527.

6 Truchuelo M, Vano-Galvan S, Pérez B, et al: Unilateral taxane-induced onychopathy in a patient with a brain metastasis. Dermatol Online J 2009; 15:7.

7 Almeida da Cruz L, Gehm Hoff PM, Seabra Ferrari CL, Pimenta Riechelmann RS: Unilateral hand-foot syndrome: does it take sides? Case report and literature review. Clin Colorec Cancer 2012;11:82-84.

$>8$ Disel U, Gürkut O, Abah H, Kaleagast H, Mertsoylu H, Ozyilkan O, Wasif Saif M: Unilateral hand-foot syndrome: an extraordinary side effect of capecitabine. Cutan Ocular Toxic 2010;29:140-142.
Matsuda S, Koketsu H, Hayakawa M, Nagata $\mathrm{N}$ : Unilateral capecitabine-related hand-foot syndrome. Intern Med 2015;54:2779.

10 Azimi E, Lerner EA, Elmariah SB: Altered manifestations of skin disease at sites affected by neurological deficit. Br J Dermatol 2015; 172:988-993.

11 Wang TS, Tsai TF: Psoriasis sparing the lower limb with post poliomyelitis residual paralysis. Br J Dermatol 2014;171:429-430.

12 Veale D, Farrell M, Fitzgerald O: Mechanism of joint sparing in a patient with unilateral psoriatic arthritis and a longstanding hemiplegia. Br J Rheumatol 1993;32:413-416.

13 Haneke E: Psoriasis in Autotransplantaten. Dermatol Mschr 1973;158:227-229.

14 Wasner G, Hilpert F, Schattschneider J, et al: Docetaxel-induced nail changes - a neurogenic mechanism: a case report. J Neuro-Oncol 2002;58:167-174. 